\title{
A multicentre study evaluating the effects of the Swedish ACE programme
}

Marie Öberg

The self-archived postprint version of this journal article is available at Linköping University Institutional Repository (DiVA):

http:/ / urn.kb.se/ resolve?urn=urn:nbn:se:liu:diva-143749

N.B.: When citing this work, cite the original publication.

This is an electronic version of an article published in:

Öberg, M., (2017), A multicentre study evaluating the effects of the Swedish ACE programme,

International J ournal of Audiology, 56(11), 876-886.

https:// doi.org/ 10.1080/ 14992027.2017.1346304

Original publication available at:

https:/ / doi.org/ 10.1080/ 14992027.2017.1346304

Copyright: Taylor \& Francis (STM, Behavioural Science and Public Health Titles)

http:// www.tandf.co.uk/journals/ default.asp 
A multicentre study evaluating the effects of the Swedish ACE programme

Running head: Öberg Effects of the Swedish ACE programme

\title{
Öberg, Marie
}

1. Division of Neuro and Inflammation Science, Department of Clinical and Experimental Medicine, Linköping University, Department of Otorhinolaryngology in Linköping, Anaesthetics, Operations and Specialty Surgery Centre, Region Östergötland, Sweden

2. The Swedish Institute for Disability Research, Linnaeus Centre HEAD, Department of Behavioural Sciences and Learning, Linköping University, Sweden.

Corresponding author:

Marie Öberg, Technical Audiology, Department of Clinical and Experimental Medicine, Linköping University, Linköping SE-581 85

E-mail: marie.oberg@liu.se

\begin{abstract}
Objective: This study investigated the effects of a modified Swedish version of an interactive group education programme, the Active Communication Education (ACE) programme, in five Swedish regions. This study also explored whether the pre- and post-programme outcomes differed with regard to region, age, gender, hearing loss (HL) or the attendance of significant others (SOs).

Design: An intervention study with between- and within-group measurements was applied.

Study Sample: Seventy-seven individuals with hearing impairments and a mean age of 73.9 years ( $\mathrm{SD}=9.8)$ from five different regions in Sweden participated in this study.
\end{abstract}


Results: Statistically significant short- and long-term effects on communication strategy use, activity, and participation were observed. The ACE programme was most effective for older individuals, women and participants with more severe HL. Individuals who attended with an SO tended to use better communication strategies. No regional differences were observed. The qualitative results, indicated that the programme increased individuals' ability to cope and restored their social identities.

Conclusions: The ACE programme is effective, is recommended for implementation in clinical settings and is considered an alternative or additional treatment to hearing aid rehabilitation. Additional studies that include younger individuals and a control group are recommended.

\section{Keywords}

Group audiological rehabilitation, communication strategies, hearing loss, activity and participation, treatment effectiveness

\section{Abbreviations}

$\mathrm{HL}=$ hearing loss

ACE $=$ Active Communication Education programme

$\mathrm{SO}=$ significant other

PTA=pure-tone average

CSS $=$ Communication Strategies Scale

HADS=Hospital Anxiety and Depression Scale

COSI=Client-Oriented Scale of Improvement

HHIE=Hearing Handicap Inventory for the Elderly

IOI-AI=International Outcome Inventory for Alternative Interventions 


\section{Introduction}

Audiological rehabilitation aims to improve communication abilities as well as reduce activity limitations and participation restrictions. Untreated hearing loss (HL) combined with reduced communication abilities can lead to social isolation, poor psychosocial health and decreased quality of life (Bridges \& Bentler, 1998; Arlinger, 2003; Öberg et al, 2012). Group audiological rehabilitation programmes effectively improve activity, participation and communication strategy use (Preminger, 2003; Hawkins, 2005; Hickson et al, 2007a; Preminger, 2007; Preminger \& Yoo, 2010). Few studies have specified assessments for certain factors such as age, gender and significant other (SO) attendance (Preminger, 2007). Hickson et al. (2007a) evaluated the Active Communication Education (ACE) programme and reported significant short- and long-term improvements in activity, participation, communication abilities and psychological well-being; however, no relationships with age, gender, better ear pure-tone average (PTA) or SO attendance were observed. Preminger (2003) did not find a correlation between reductions in activity and participation and degree of HL or age; rather, this author found improvements in activity and participation among individuals who attended the rehabilitation programme with an SO.

The ACE programme uses a problem-solving interactive approach that encourages participants to discuss their communication difficulties with group members and facilitators as well as practise communication solutions. The primary aims of the ACE programme are to reduce communication difficulties in everyday life and improve quality of life (Hickson et al, 2007b). The ACE programme was previously translated into Swedish and used in two studies. After a pilot study that only included participants who were 87 years old, the content of the original programme was modified (Öberg et al, 2014a, 2014b). 
An evaluation of the Swedish version of the ACE programme revealed significant long-term effects regarding communications strategies, activity, participation, depression and anxiety (Öberg et al, 2014a). Significant differences in the effects of age, gender, hearing aid users/nonusers and the degree of HL were observed. Women exhibited improvements in their short- and long-term use of communication strategies and improved their long-term activity, participation and psychosocial health. Individuals with mild HL and older participants improved their short- and long-term use of communication strategies. Individuals with moderate HL improved their long-term psychosocial health.

Our earlier ACE studies were conducted at the clinic in Linköping and were administered by the same staff. The procedure and guidelines for audiological rehabilitation vary between regions. A multicentre study was conducted to increase the generalizability and to further investigate the effects of the ACE programme and the groups that benefit most from this kind of audiological rehabilitation. A multicentre study includes a larger number of participants with different geographic locations and could generate a wider range of population groups, which might increase the generalizability of the results of the Swedish ACE programme.

The current study investigated the effects of the Swedish version of the ACE programme in five regions. Specifically, this study investigated 1) the pre- and postprogramme (i.e., short- and long-term) outcomes for communication strategy use, activity, participation, anxiety and depression; 2) whether pre- and post-programme outcomes differed among groups with regard to region, age, gender, degree of HL, and the presence of an SO; and 3) post-programme qualitative assessments. 


\section{Methods}

\section{Participants}

Between 2013 and 2014, 102 participants were recruited to participate in the ACE programme. Participants from five Swedish regions, Linköping ( $n=22)$, Västerås $(n=20)$, Uppsala (n=16), Falun (n=16) and Örebro (n=3), were included. In total, 17 groups were included. Fourteen groups included seniors (age $\geq 66$ years), and 3 groups included workingage participants (age $\leq 65$ years).

The participants were recruited from each clinic by audiologists and hearing therapists and via poster advertisements in the waiting rooms. Clinically referred individuals who experienced subjective communication difficulties because of HL, including individuals who did not use hearing aids (nonusers), were eligible to participate. The lower limit for hearing loss was a PTA $(0.5,1.0,2.0$ and $4.0 \mathrm{kHz})>25 \mathrm{~dB} H L$ for the worse ear but no upper PTA limits were defined for the participant inclusion criteria.

Sixty-two women and 40 men with a mean age of 73.9 years (SD)=10.2 years; range=41-94 years) and a PTA of 55.1 dB $\mathbf{H L}(\mathrm{SD}=21.2)$ in the better ear were recruited. Of these $\mathbf{1 0 2}$ participants, 92 completed the ACE program, whereas 7 never started the program, and 3 only attended one session and left the intervention because of an illness. As suggested by Hickson et al. (2007a), only participants who attended 3 or more sessions were included in the analyses of the effects of the programme. Of the 92 participants, 83 completed the questionnaires after 3 weeks, and 77 participants completed the questionnaires after 6 months. This study only includes the data from the 77 participants who completed the questionnaires at all three time points. Table 1 presents the demographic data of the 77 participants.

Most of the subjects participated in five sessions (66\%). Thirty percent participated in 4 sessions, and 4 percent participated in 3 sessions. Nineteen (25\%) participants brought an 
SO to the sessions. The majority of the significant others (SOs) participated four (32\%) or five $(63 \%)$ times.

\section{Table 1 here}

\section{Outcome measures}

Pre- and post-programme measures

Validated questionnaires that have been previously used in Sweden and in an earlier ACE study were used in the present study (Öberg et al, 2007, 2008, 2009; Thorén et al, 2011; Öberg et al, 2014a; Thorén et al, 2014). The Hearing Handicap Inventory for the Elderly (HHIE) assesses the effects of HL among elderly people (Ventry \& Weinstein, 1982). This questionnaire consists of 25 items and is divided into two subscales. Thirteen items explore the emotional consequences of HL (HHIE E), and 12 items explore the social and situational effects of HL (HHIE S). Three response options exist for each item: yes (4), sometimes (2), or no (0). Higher scores represent greater perceived activity limitations and participation restrictions.

The Communication Strategies Scale (CSS) is a part of the Communication Profile for the Hearing Impaired (Demorest \& Erdman, 1987). The CSS has 25 items and is divided into three subscales: maladaptive (M), verbal (V) and nonverbal (NV). Hence, the CSS assesses both adaptive and maladaptive communication strategies. Responses are rated on a five-point scale from almost never (1) to almost always (5). Higher scores indicate fewer problems.

The Hospital Anxiety and Depression Scale (HADS; Zigmond \& Snaith, 1983) assesses the presence/absence of symptoms of anxiety and depression among medical outpatients. The HADS consists of 14 items, each of which has four response choices (0-3) with subscales for anxiety and depression. Higher scores indicate more symptoms (for a detailed description of the questionnaire, see Öberg et al., 2014a). 
A modified version of the Client-Oriented Scale of Improvement (COSI; Dillon et al, 1997; Hickson et al, 2007a) was used to evaluate the extent to which the individual goals of the programme were achieved. The participants themselves identified their individual goals for the programme and assessed these goals after the programme by selecting from the following options: worse (1), no different (2), slightly better (3), better (4), or much better (5).

The International Outcome Inventory for Alternative Interventions (IOI-AI) is a seven-item questionnaire that evaluates the effect of interventions such as the ACE programme (Noble, 2002). Each item represents a different outcome domain: the daily use of ACE strategies (Use), benefits, residual activity limitations (RAL), satisfaction with the programme, residual participation restrictions (RPR), impact on others (IoO) and quality of life (QoL). Each item has five response alternatives that differ depending on the items; higher scores indicate better outcomes.

The open-ended feedback questions, "What did you like about the sessions?" and “How could the sessions be improved?" were answered 3 weeks after the participants' completed the ACE programme.

\section{Procedure}

The study was approved by the regional ethics committee. Each clinic invited people with hearing difficulties to participate in the ACE programme. The author mailed the HHIE, HADS, CSS and the informed consent forms to the referred individuals who agreed to participate in the evaluation to allow them to complete the forms at home before the first session of the ACE programme.

The Swedish ACE programme consists of five weekly two-hour sessions, and the optimal group size is 6 to 10 participants. The first session starts with a group goal- 
setting activity in which the participants expressed the issues they were particularly interested in addressing during the programme. In the Swedish ACE programme, the four additional modules include tasks and discussions related to understanding conversation in background noise, my ear and my hearing loss, how to live with a hearing loss and listening to other signals. Within each session, participants discuss their communication activities, possible solutions and practical exercises for communication strategies (for a detailed description of the procedure used in the sessions, see Hickson et al., 2007b). The sessions occurred in the clinic. Three weeks after the programme was completed, the participants responded to the same questionnaires that they initially completed as well as the IOI-AI, the COSI and the open-ended feedback question.

To investigate the long-term effects of the program, a follow-up assessment sent via post was conducted 6 months after the ACE programme was completed. The same questionnaires (the HHIE, HADS, CSS, and IOI-AI but not the COSI), which were administered 3 weeks after the program, were mailed to the participants. An additional item regarding whether the participants had taken any action (i.e., implemented any new communication strategies) as a result of attending the programme was included (Hickson et al, 2007a).

\section{Data analyses}

Pre- and post-programme (i.e., short- and long-term) outcomes for communication strategy use, activity, participation, anxiety and depression were analysed using a repeated-measures one-way analyses of variance (ANOVA). Statistically significant effects were examined using Bonferroni-corrected post hoc t-tests. To investigate the pre- and post-programme outcomes for different factors, separate ANOVAs were performed with a between-group factor for region (4), age ( $\leq 65$ vs. $\geq 66$ years old), gender, degree of HL (mild, moderate, 
severe, or profound), and the presence of SO with a within-group factor of time (pre, after 3 weeks and after 6 months) and between- and within-group interactions. When analysing the data related to region, Örebro was deleted from the data analysis because of a small number of participants. Quantitative data were analysed using STATISTICA (Statsoft, 2009, version 13), and results with $p$-values $<.05$ were considered statistically significant. The comments providing feedback on the programme content and the COSI goals were entered in Microsoft Excel and analysed using qualitative content analyses (Graneheim \& Lundman, 2004). The codes were categorized into themes and categories by the author.

\section{Results}

Pre- and post-ACE programme outcomes

To investigate the short- and long-term effects, a repeated-measures ANOVA was performed to compare the pre-, 3-week and 6-months assessments.

Statistically significant interaction effects were examined using Bonferronicorrected post hoc t-tests for the assessments performed before and 3 weeks after the programme (1-2) or 3 weeks and 6 months after the programme (2-3).

Table 2 presents the means, SDs, ranges, and F-values for the different questionnaires. As this table shows, significant long-term within-group effects were identified with regard to communication strategy use (CSS tot), verbal communication strategies (CSS V), activity and participation (HHIE tot, HHIE S, HHIE E) for pre- vs. 6-month postprogramme comparisons. Post hoc measurements revealed significant short-term improvements (1-2) for communication strategy use, verbal communication strategies, activity and participation (HHIE tot, HHIE E). Statistically significant improvements in activity and participation in HHIE S were identified between the 3-week and 6-month (2-3) post-programme assessments. Significant within-group effects (3 weeks vs. 6 months post 
program) were identified with regard to the IOI-AI total score and item 4 on the IOI-AI, such that the total score and satisfaction deteriorated over time.

$>$ Table 2 here $<$

\section{Pre- and post-ACE programme outcomes for the different groups}

A series of ANOVAs were performed to investigate the short- and long-term effects of the programme over different factors (region, age, gender, degree of HL, and the presence of a SO). The statistically significant F-values for the interaction and between-group and withingroup effects are presented in Table 3, and the means and SDs for the statistically significant outcomes between groups are presented in Table 4.

\section{Effects of age}

Significant interaction effects of age were identified with regard to HHIE tot and HHIE S. The post hoc t-tests were significant for HHIE tot and HHIE S among older participants ( $\geq 66$ years), revealing short- (HHIE tot, $p<.01$; HHIE $S, p<.05$ ) and long-term (HHIE tot, $p<.001$; HHIE S, $p<.001$ ) improvements in activity and participation; however, the scores for the younger participants were unchanged. Also statistically significant between-group effects on HHIE tot (no significant differences after post hoc test) and HHIE E were found, and the post hoc test revealed significant short- and long-term improvements among older individuals; however, the younger group did not show significant changes (Table 4). Age effects were also identified for CSS tot; however, the post hoc test did not reveal differences between groups. Statistically significant effects of time were identified for CSS tot and CSS V, as post hoc analyses revealed that older individuals improved their use of verbal strategies over a brief period of time $(p<.05)$ (Table 3). 


\section{Effects of gender}

No significant interaction, between-group or short-term, effects were identified.

Statistically significant long-term effects for CSS tot and CSS V were identified; however, the post hoc test did not show significant between-group differences. Long-term effects were also found for HHIE tot, HHIE E and HHIE S and the post hoc tests revealed significantly improved scores for HHIE tot $(p<.001)$, HHIE E $(p<.01)$, and HHIE S $(p<.01)$ among women and significant improvements in HHIE tot $(p<.05)$ among men (Table 3).

\section{Effects of degree of hearing loss}

Statistically significant interaction effects of HL on HHIE tot and HHIE S were identified. In addition, significant between-group effects were found for HHIE tot and HHIE S, and the post hoc tests revealed significant differences between the initial score of the mild HL group compared with those with profound HL (HHIE tot, $p<.05$; HHIE S, $p<.01$ ). The initial scores varied from 38.0 points for mild HL to 60.0 points for profound HL (Table 4). Significant between-group effects were identified for HHIE E (no significant differences post hoc). Significant between-group effects were also identified with regard to the IOI-AI (no significant differences post hoc) and for CSS V. Post hoc tests initially revealed significant differences between mild and profound HL $(p<.05)$; the former group used significantly fewer verbal strategies than the latter group (Tables 3 and 4). Long-term effects on HHIE tot, HHIE E, and HHIE S were found and post hoc tests revealed significant improvements for individuals with profound $H \mathbf{H}$, whereas improvements for the other groups were not statistically significant (Tables 3 and 4 ).

\section{Effects of region}


No interaction or main effects of region were identified. Significant effects of time on CSS tot, CSS V, HHIE tot, HHIE E and HHIE S were found; however, post hoc tests did not reveal between-group differences.

\section{Effects of SO}

Significant interaction effects regarding SO attendance were identified with regard to the IOIAI tot. Individuals who brought their SOs exhibited improved long-term scores, whereas those who did not exhibited reduced scores. However, a post hoc test did not reveal betweengroup differences. To further investigate the effects for the items of the IOI-AI, separate analyses were performed on each item. The same pattern was observed for item 1 (daily use of ACE strategies, Fig 1) and item 5 (residual participation restriction). However, a post hoc test did not reveal differences. Long-term effects on HHIE tot, HHIE E, and HHIE S were found, where post hoc tests revealed significant improvements for both groups with regard to HHIE tot (with SO, $p=.025$ : without SO, $p=.0007$ ) and significant improvements for the group without SOs were observed on two subscales (HHIE E, $p=.004$; HHIE S, $p=.0013$ ). Long-term improvements were also identified for CSS V, but the post hoc test did not show between-group differences (Tables 3 and 4).

Tables 3 and 4 and Fig 1 here

\section{Qualitative assessments}

COSI

The individual COSI goals of the programme were assessed 3 weeks after the ACE programme was completed. In total, 70 participants generated 245 goals. Not all goals were relevant to the ACE programme, and 212 goals remained after irrelevant goals were excluded (Table 5). Excluded irrelevant goals were more general goals and were not specifically related to the intervention, such as participants who liked to have more 
broadcasts without background music or more public premises with inductive loops. The participants assessed their goals after the programme by selecting from the options from worse (1) to much better (5). The overall mean score for these goals was 3.49 $(\mathrm{SD}=1.04)$, indicating that participants described the improvements in skills related to their goals as slightly better to better. The goals were categorized into 7 themes: learn communication skills, learn about hearing aids and assistive listening devices, meet in a group to share experiences, learn more about my HL, prevent isolation and increase selfconfidence, learn how to hear better on the telephone and learn how to hear better when listening to the TV and in the car. Most goals were categorized as "learning communication skills” (87). The goals related to "prevent isolation and increase self-confidence” improved the most (mean=4.07, $\mathrm{SD}=1.00$ ), and the goals related to "Learn how to hear better on the telephone” exhibited the least improvement (mean=3.12, SD=1.13). Examples of goals related to the category "prevent isolation and increase self-confidence” included "want to have courage to tell other people I have HL" and "trying to face my fear regarding conversations with strangers”.

Insert Table 5 here

Feedback item: What did you like about the sessions?

After completing the course, the participants evaluated what they liked about the programme. In total, 175 comments were generated. These comments were categorized into 9 categories. The 4 most common categories included learning from the group (27\%), structure of the programme (19\%), general positive comments (14\%) and positive comments about the facilitators (10\%). Participants appreciated learning more about their HL, and comments were related to learning more about how to interpret audiograms (8\%). The remaining categories were learning communication strategies (7\%), increased knowledge about hearing assistive 
devices (6\%), the atmosphere of the group sessions (5\%) and comments related to increased self-confidence (4\%). The participants provided many examples of how they learned from the group. One such example is "I concluded that we all described similar problems and got suggestions from the others on how to cope with them".

\section{Feedback item: How could the sessions be improved?}

Eighty-two comments were received. The most common answer was that there was nothing to improve (26\%), followed by learning more about hearing aids and assistive devices (17\%) and a need for more sessions (10\%).

Action taken as a result of attending the programme

Approximately half of the participants did not answer the question regarding what actions the participants had taken after the ACE programme was completed at the 6-month follow-up or answered they had not taken any actions. Of the 40 comments, the most common were categorized as "telling others about my HL more often” and “choose where to sit more often”. One comment regarding telling others about the HL was: “I acknowledge that I do not hear; previously, I did not say anything and pretended to hear”. Many participants also commented that they had "used new hearing aids or assistive devices more often”.

\section{Discussion}

The current study evaluated the effects of the Swedish version of the ACE programme in five regions. Statistically significant short- and long-term effects on communication strategy use, activity and participation were identified. The programme seems effective irrespective of any regional differences in hearing health care. The programme was most effective for older individuals, women and participants with more severe HL. 
Short- and long-term effects on communication strategy use (Chisolm et al, 2004; Kramer et al, 2005; Hickson et al, 2007a; Öberg et al, 2014a) and activity and participation (Hawkins, 2005; Hickson et al, 2007a; Preminger \& Yoo, 2010) have been observed in previous intervention studies. Overall, the participants in the current study improved their HHIE scores by 7 points. Other intervention studies have reported improvements of 6 to 20 points (Preminger, 2003; Preminger \& Yoo, 2010; Thorén et al, 2014, 2015). However, significant variations in improvement were related to the degree of HL, as participants with profound HL showed improvements of 17 points.

In the present study, significant differences in activity, participation and communication strategy use were identified with regard to age and degree of HL and in activity and participation with regard to gender. This finding contradicts the findings from the study by Hickson et al (2007a). Although the intervention is the same, comparisons of the results are difficult because the outcome measures differ between the studies. An overview of statistically significant overall and between-group outcomes for ACE studies is presented in Table 6.

Insert Table 6 here

Short- and long-term improvements in activity and participation were identified for both older and younger individuals; however, the effects were only statistically significant for older individuals. Other studies did not identify age-related differences in activity and participation. (Beynon et al, 1997; Preminger, 2003; Hickson et al, 2007a; Öberg et al, 2014a). Improvements in verbal strategies were identified for both older and younger individuals; however, the effects were only statistically significant for older individuals. This result was not in line with the results in Jansson et al, (2002), finding younger participants to increase verbal strategies. The statistically significant improvement in verbal strategies 
only among older individuals may be related to the low statistical power for younger individuals, because they only represented $14 \%$ of the present sample.

Improvements in the HHIE varied by the degree of HL. In this study, these improvements varied from approximately 5 points for individuals with mild and moderate HL to 17 points for individuals with profound HL. An improvement of 18 to 20 points is comparable with the magnitude of an improvement of a hearing aid intervention (McArdle et al, 2005; Öberg et al, 2008, 2009). Thus, individuals with profound HL experienced a considerable benefit from the ACE programme. Individuals with profound HLs have often lived with a HL for many years and visited the hearing clinic many times to be provided with new hearing aids and assistive devices. They might have attained more knowledge about hearing and HL but have not always had an opportunity to talk about the emotional consequences of a HL. In the group sessions, the group members could express, compare and confirm their experiences, which could help these individuals.

Participants who brought a SO to the sessions tended to use the learned communication strategies more often than participants without SOs. Many older people do not attend social activities and are more likely to communicate at home. If their SOs have also learned to facilitate communication, then the potential for using different strategies might increase. Preminger (2003) did not observe statistically significant differences in communication strategy use between the groups who brought or did not bring a SO. The content and the outcome measures evaluating communication strategy use differed for the ACE programme and the group audiologic rehabilitation (AR) classes (Preminger, 2003). The AR classes focused on training in speechreading, auditory perception and communication strategies, whereas the ACE programme does not specifically include speech reading and auditory training and instead focuses more on learning different communication strategies. The focus on learning communication 
strategies might also be more relevant for the $\mathrm{SO}$, and more time spent on this area could have improved the use and the benefit of the strategies at home. According to the study by Preminger (2003), participants who brought a SO exhibited significant improvements in activity and participation measured with the HHIE questionnaire. In the present study, no significant differences in HHIE total were observed between the groups that brought or did not bring a SO, as both groups improved. However, the group that brought a SO exhibited a 9-point improvement compared to the approximately 6-point improvement for the group without a SO. Only $25 \%$ of the participants in the present study had SOs who attended. It is preferable more SOs attend the sessions in the ACE programme. During the sessions, many SOs expressed that they better understood the consequences of HL when they heard other participants discuss their hearing problems and the strategies they used.

No differences between regions were identified. Thus, the programme was effective, regardless of the geographic locations, facilitators and differences in how the audiological rehabilitation is organized. The highest improvement in individual goals occurred for the category "prevent isolation and increase self-confidence” (mean=4.07). These different goals were related to being more open about acknowledging the HL to others and being braver when talking to strangers. These goals are consistent with the most common comments concerning the type of actions that the participants had taken after the ACE programme. These goals represent an important effect of the programme because they likely prevent isolation.

When describing what the participants liked most about the program, it was found that "learning from the group" was the most common category. This result might have resulted in the greatest improvements regarding the COSI goals related to preventing isolation and increasing self-confidence. These qualitative results indicate the effectiveness of 
using a problem-solving strategy to encourage people with hearing impairments to use different communication strategies. The problem-solving strategy used in the ACE programme enables discussions in which participants provide examples of their coping strategies and emotions regarding HL. Participants reported that the group had a positive atmosphere, which allowed them to share their feelings. The ACE programme offers additional opportunities for participants to discuss their feelings and aspects related to HL, as the hearing aid-fitting procedure does not often provide information about the emotional consequences of HL (Barker et al, 2016). Hétu (1996) claimed that group settings effectively restore social identities because individuals are able to clarify their identities by sharing their frustrations and difficulties with each other.

When evaluating the benefits of the ACE program, it is important to consider both quantitative and qualitative assessments because it is difficult to measure all aspects using standardized questionnaires. As shown in the study by Preminger (2007), people reported that the group rehabilitation programme was very helpful, and the participants developed the courage to admit their HL, even if the scores for the questionnaire measuring activity and participation did not change.

When comparing the data from the present study of participants from 5 regions in Sweden with an earlier Swedish study that included participants only from one region, many similarities and some differences were noted (Öberg et al, 2014a). First, differences regarding demographic data were observed. The participants in the present study experienced greater HL (PTA: 57 dB HL vs. 42 dB HL); thus, more individuals had severe-to-profound HL in the current study. The current study included older individuals (mean age: 74 years old vs. 69 years old) and fewer nonusers (5\% vs. 24\%). Differences were found with regard to activity, participation and mental health. In the present study, individuals with a more severe HL exhibited the greatest improvements in activity and participation, whereas only 
individuals with a mild HL improved in the earlier study (Table 6). The reason for the greater improvement for the group with a profound HL may be linked to the high initial scores for this group. The earlier study did not generate comparable data for individuals with a profound HL, as only 2 individuals reached the limit for severe HL. No significant improvements were found with regard to mental health in the present study compared with the earlier study in which individuals with moderate HL exhibited long-term improvements. The lack of improvement in mental health observed in this study might be related to the better initial scores of the current individuals. In the present study, women exhibited improved activity and participation scores. The earlier ACE study revealed more differences between men and women; specifically, women had significant improvements in communication strategies and mental health.

The similarities between the studies were related to improvements in communication strategy use and activity and participation. Similarities were found with regard to improvements in communication strategies among older individuals and improvements in activity and participation among women. In both studies, those with mild HL initially exhibited a reduced use of communications strategies. This effect was found for verbal strategies in the present study, whereas this effect was found for nonverbal strategies in the previous study. These results might be explained by the fact that individuals with milder HL most likely do not need to use these strategies as often as those with more severe HL. The studies also exhibited similarities in the qualitative assessments: learning communication strategies and learning from the group were the most beneficial outcomes, and the most common action taken after the programme was being more open about telling others about their HL.

The limitations of this study include the absence of a control group as well as the unequal and limited numbers of participants in each group. When the variables were 
divided into four subgroups, the number of participants in each group sometimes became small with difficulties to achieve statistically significant between-group effects.

\section{Conclusions}

The results from this multicentre study showed statistically significant short- and longterm effects on communication strategy use, activity and participation but no improvements in anxiety and depression. The improvements in communication strategy use and activity and participation are consistent with the results of the previous Swedish ACE study in which all of the participants were recruited from the same clinic (Öberg et al, 2014a). The ACE programme was most effective for older individuals, women and participants with a more severe HL, and individuals who attended the sessions with SOs tended to use better communication strategies. No differences between regions were identified.

The problem-solving strategy used in the ACE programme provides participants with additional opportunities to discuss their feelings and other aspects related to HL. Based on the current qualitative results, this programme seems to increase individuals' ability to cope and restores their social identities. The ACE programme is recommended for implementation in clinical settings and is considered an alternative or additional treatment to traditional hearing aid rehabilitation. Future studies with younger individuals and a control group are recommended. Future studies should pay closer attention to the advantages of bringing a SO and to the SOs experiences of the programme. 


\section{References}

Arlinger, S. 2003. Negative consequences of uncorrected hearing loss-a review. Int J Audiol, 42 Suppl. 2, 2S17-2S20.

Barker, F., Mackenzie, E. \& de Lusignan, S. 2016. Current process in hearing-aid fitting appointments: An analysis of audiologists' use of behaviour change techniques using the behaviour change technique taxonomy (v1). Int J Audiol, 55, 643-652.

Beynon, G.J., Thornton, F.L. \& Poole, C. 1997. A randomized, controlled trial of the efficacy of a communication course for first time hearing aid users. Br J Audiol, 31, 345-351.

Bridges, J.A. \& Bentler, R.A. 1998. Relating hearing aid use to well-being among older adults. Hear J, 51, 39-44.

Chisolm, T.H., Abrams, H.B. \& McArdle, R. 2004. Short- and long-term outcomes of adult audiological rehabilitation. Ear Hear, 25, 464-477.

Demorest, M.E. \& Erdman, S.A. 1987. Development of the communication profile for the hearing impaired. J Speech Hear Disord, 52, 129-143.

Dillon, H., James, A. \& Ginis, J. 1997. Client oriented scale of improvement (COSI) and its relationship to several other measures of benefit and satisfaction provided by hearing aids. J Am Acad Audiol, 8, 27-43.

Graneheim, U.H. \& Lundman, B. 2004. Qualitative content analysis in nursing research: concepts, procedures and measures to achieve trustworthiness. Nurse Educ Today, 24, 105-112.

Hawkins, D.B. 2005. Effectiveness of counseling-based adult group aural rehabilitation programs: A systematic review of the evidence. J Am Acad Audiol, 16, 485-493.

Hétu, R. 1996. The stigma attached to hearing impairment. Scand Audiol Suppl, 43, 12-24. 
Hickson, L., Worrall, L. \& Scarinci, N. 2007a. A randomized controlled trial evaluating the active communication education program for older people with hearing impairment. Ear Hear, 28, 212-230.

Hickson, L., Worrall, L. \& Scarinci, N. 2007b. Active Communication Education (ACE): A Program for Older People with Hearing Impairment. Brackley, UK: Speechmark. Jansson, G., Trulsson, U., Wiberg, A. \& Hallberg, L.R. 2002. Group rehabilitation of patients with acquired hearing impairment and their close relatives: Evaluation of short- and long-term effects. Scand J Disabil Res, 4, 43-57.

Kramer, S.E., Allessie, G.H., Dondorp, A.W., Zekveld, A.A. \& Kapteyn, T.S. 2005. A home education program for older adults with hearing impairment and their significant others: A randomized trial evaluating short- and long-term effects. Int J Audiol, 44, 255-264.

McArdle, R., Chisolm, T.H., Abrams, H.B., Wilson, R.H. \& Doyle, P.J. 2005. The WHODAS II: Measuring outcomes of hearing aid intervention for adults. Trends Amplif, 9, 127-143.

Noble, W. 2002. Extending the IOI to significant others and to non-hearing-aid-based interventions. Int J Audiol, 41, 27-29.

Öberg, M., Andersson, G., Wänström, G. \& Lunner, T. 2008. The effects of a sound awareness pre-fitting intervention: A randomized controlled trial. Audiol Med, 6, 129140.

Öberg, M., Andersson, G., Wänström, G. \& Lunner, T. 2009. The effects of a pre-fitting intervention on hearing aid benefit: A randomized controlled trial. Audiol Med, 7, 211-225. 
Öberg, M., Bohn, T. \& Larsson, U. 2014a. Short- and long-term effects of the modified Swedish version of the active communication education (ACE) program for adults with hearing loss. J Am Acad Audiol, 25, 848-858. doi:10.3766/jaaa.25.9.7.

Öberg, M., Bohn, T., Larsson, U. \& Hickson, L. 2014b. A preliminary evaluation of the active communication education program in a sample of 87-year-old hearing impaired individuals. J Am Acad Audiol, 25, 219-228. doi:10.3766/jaaa.25.2.10.

Öberg, M., Lunner, T. \& Andersson, G. 2007. Psychometric evaluation of hearing specific self-report measures and their associations with psychosocial and demographic variables. Audiol Med, 5, 188-199.

Öberg, M., Marcusson, J., Nägga, K. \& Wressle, E. 2012. Hearing difficulties, uptake, and outcomes of hearing aids in people 85 years of age. Int $J$ Audiol, 51, 108-115.

Preminger, J.E. 2003. Should significant others be encouraged to join adult group audiologic rehabilitation classes? J Am Acad Audiol, 14, 545-555.

Preminger, J.E. 2007. Issues associated with the measurement of psychosocial benefits of group audiologic rehabilitation programs. Trends Amplif, 11, 113-123.

Preminger, J.E. \& Yoo, J.K. 2010. Do group audiologic rehabilitation activities influence psychosocial outcomes? Am J Audio, 19, 109-125.

Statsoft. 2009. STATISTICA Inc. (data analysis software system) Version 13. Available at www.statsoft.com.

Thorén, E.S., Öberg, M., Andersson, G. \& Lunner, T. 2015. Internet interventions for hearing loss. Am J Audiol, 24, 316-319.

Thorén, E.S., Öberg, M., Wänström, G., Andersson, G. \& Lunner, T. 2014. A randomized controlled trial evaluating the effects of online rehabilitative intervention for adult hearing-aid users. Int J Audiol, 53, 452-461. 
Thorén, E., Svensson, M., Törnqvist, A., Andersson, G., Carlbring, P. \& Lunner, T. 2011. Rehabilitative online education versus Internet discussion group for hearing aid users: A randomized controlled trial. J Am Acad Audiol, 22, 274-285.

Ventry, I.M. \& Weinstein, B.E. 1982. The hearing handicap inventory for the elderly: A new tool. Ear Hear, 3, 128-134.

Zigmond, A.S. \& Snaith, R.P. 1983. The hospital anxiety and depression scale. Acta Psychiatr Scand, 67, 361-370.

\section{Acknowledgements}

The author thanks all of the participants. The author also thanks the entire staff involved in this project: Bodil Eriksson, Therese Bohn-Eriksson, Britt-Marie Fasth-Knudsen, Lena Andersson, Annica Samuelsson, Eva Matthiesen, Maria Gustavsson, Kajsa Hellström, Kerstin Kans and Malin Andersson. Grants from the Swedish Association of Hard of Hearing People and the Östergötland County Council Research Foundation supported this study.

\section{Declaration of interest:}

The author has no potential conflicts of interest to declare. 


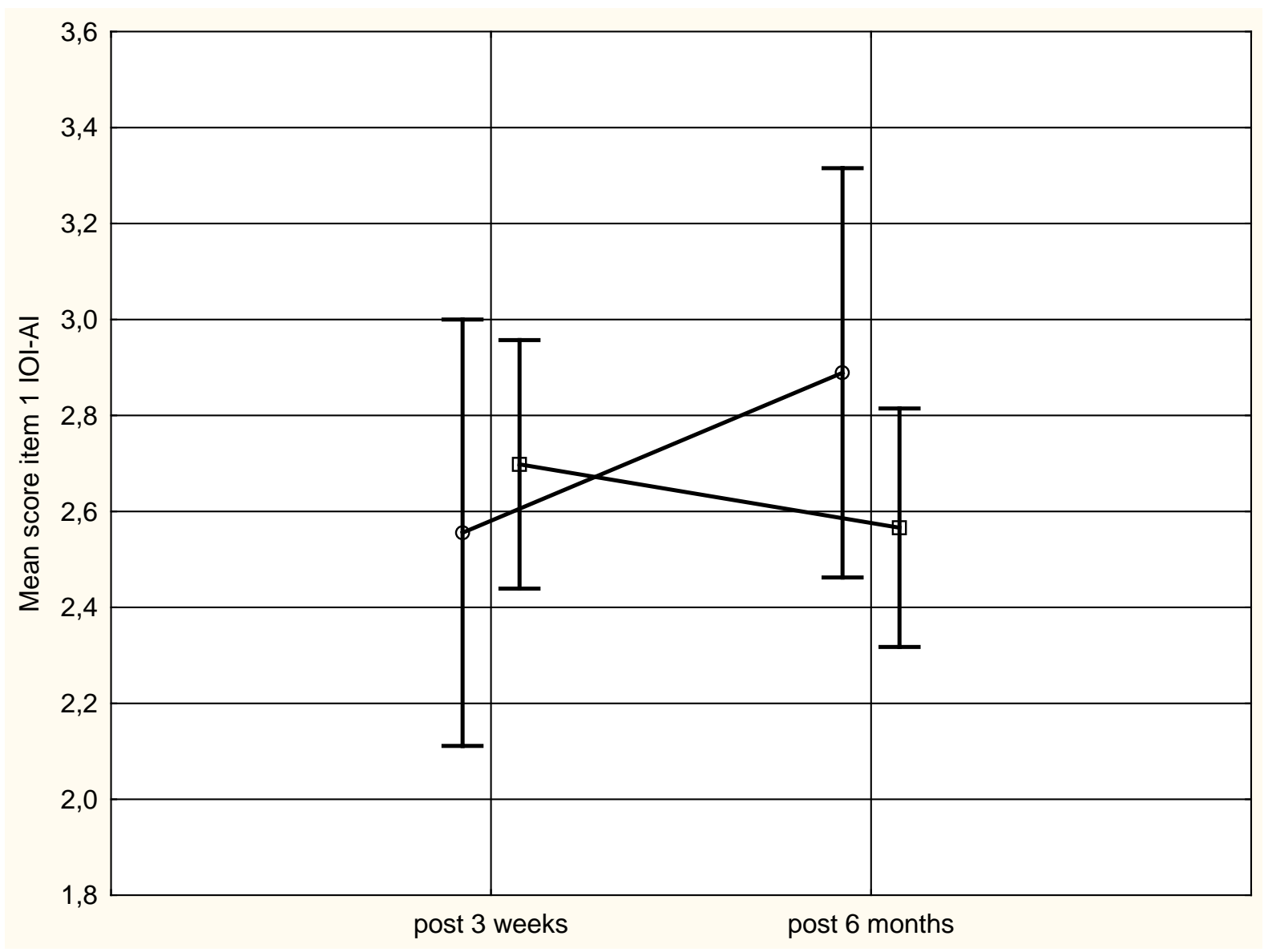

Figure 1. Means scores and 95\% confidence intervals for IOI-AI item 1, daily use of ACE strategies, for the group who brought (circles) SO and the group who did not bring SO (squares). 
Table 1. Means, SDs and range for demographic data

\begin{tabular}{|c|c|c|}
\hline \multicolumn{3}{|l|}{ Variable } \\
\hline \multirow[t]{3}{*}{ Age } & Mean & 73.9 \\
\hline & SD & 9.8 \\
\hline & Range & $41-94$ \\
\hline Age $\leq 65$ year & Number (\%) & $11(14 \%)$ \\
\hline Age $\geq 66$ year & Number (\%) & $66(86 \%)$ \\
\hline \multirow[t]{3}{*}{ PTA $^{\mathrm{a}}$ better ear } & Mean & 57.2dB HL \\
\hline & SD & 21.2dB HL \\
\hline & Range & 17.5-100dB HL \\
\hline Mild HL ${ }^{\mathrm{b}}$ (25-40dB HL) & Number (\%) & $18(23 \%)$ \\
\hline Moderate HL (41-60dB HL) & Number (\%) & 25 (33\%) \\
\hline Severe HL (61-80dB HL) & Number (\%) & $26(34 \%)$ \\
\hline Profound HL (>81dB HL) & Number (\%) & $8(10 \%)$ \\
\hline \multirow[t]{2}{*}{ Gender } & Women & $47(61 \%)$ \\
\hline & Men & $30(39 \%)$ \\
\hline Attended SO ${ }^{c}$ & Number (\%) & $19(25 \%)$ \\
\hline \multicolumn{3}{|l|}{ Hearing devices } \\
\hline Nonuser & Number (\%) & $4(5 \%)$ \\
\hline Hearing aid user & Number (\%) & $68(88 \%)$ \\
\hline $\mathrm{CI}^{\mathrm{d}}$-user & Number (\%) & $5(7 \%)$ \\
\hline \multirow[t]{3}{*}{ Hearing aid use (years) } & Mean & 18.7 \\
\hline & SD & 15.7 \\
\hline & Range & $1-59$ \\
\hline \multirow[t]{3}{*}{ CI-use (years) } & Mean & 4.5 \\
\hline & SD & 4.9 \\
\hline & Range & $1-8$ \\
\hline \multicolumn{3}{|l|}{$\mathrm{N}=77$} \\
\hline \multicolumn{3}{|l|}{ a Pure tone average for $0.5,1,2,4 \mathrm{kHz}$} \\
\hline \multicolumn{3}{|l|}{${ }^{\mathrm{b}} \mathrm{HL}=$ Hearing Loss } \\
\hline \multicolumn{3}{|l|}{${ }^{\mathrm{c}} \mathrm{SO}=$ Significant Other } \\
\hline${ }^{\mathrm{d}} \mathrm{CI}=$ Cochlear implant & & \\
\hline
\end{tabular}




\begin{tabular}{|c|c|c|c|c|c|c|c|c|c|c|c|c|c|}
\hline \multicolumn{14}{|c|}{ Table 2. Means, SDs, Ranges and $F$ values for pre-post 6 mo (1-3). Post hoc analyses pre-post 3 wk (1-2) an post-hoc 3wk-post 6 mo (2-3) } \\
\hline Questionnaire & Mean pre & SD & Range & \begin{tabular}{|c|} 
Mean post \\
3wk
\end{tabular} & SD & Range & $\begin{array}{c}\text { Mean post } \\
6 \mathrm{mo}\end{array}$ & SD & Range & $F$-values 1-3 & $\mathrm{N}^{*}$ & Post hoc 1-2 & Post hoc 2-3 \\
\hline HHIE tot & 50.67 & 18.67 & $4-92$ & 46.6 & 18.58 & $8-86$ & 43.58 & 18.94 & $6-88$ & $F(2,150)=13.96 p>0.001$ & 76 & $p=0.0054$ & ns $(\mathrm{p}=0.11)$ \\
\hline HHIE S & 26.90 & 9.92 & $4-46$ & 25.23 & 9.71 & $4-44$ & 22.98 & 9.15 & $4-46$ & $F(2,150)=12.17, p>0.001$ & 76 & ns $(p=0.087)$ & $p=0.021$ \\
\hline HHIE E & 23.76 & 10.83 & $0-46$ & 21.36 & 10.84 & $0-46$ & 20.59 & 11.14 & $2-44$ & $F(2,150)=11.37, p>0.001$ & 76 & $p=0.0014$ & $\mathrm{~ns}(\mathrm{p}=1.0)$ \\
\hline HADS tot & 9.35 & 6.47 & $0-25$ & 9.76 & 6.46 & $0-25$ & 9.30 & 6.59 & $0-23$ & $F(2,152)=0.49 \mathrm{p}=0.61$ & 77 & & \\
\hline HADS D & 4.47 & 3.26 & $0-15$ & 4.60 & 3.06 & $0-12$ & 4.39 & 3.22 & $0-11$ & $F(2,152)=0.25 \mathrm{p}=0.78$ & 77 & & \\
\hline HADS A & 4.88 & 4.04 & $0-16$ & 5.17 & 4.12 & $0-16$ & 4.91 & 3.99 & $0-14$ & $F(2,152)=0.46 \mathrm{p}=0.63$ & 77 & & \\
\hline CSS tot & 3.54 & 0.42 & $2.76-4.64$ & 3.64 & 0.46 & $2.64-4.72$ & 3.64 & 0.43 & $2.76-4.68$ & $F(2,152)=4.10 p=0.018$ & 77 & $p=0.045$ & $\mathrm{~ns}(\mathrm{p}=1.0)$ \\
\hline CSS M & 3.74 & 0.61 & $2.44-4.67$ & 3.73 & 0.63 & $2.33-4.78$ & 3.81 & 0.68 & $1.88-4.77$ & $F(2,152)=1.04 \mathrm{p}=0.36$ & 77 & & \\
\hline CSS V & 2.89 & 0.76 & $1.5-4.87$ & 3.14 & 0.78 & $1.37-4.75$ & 3.08 & 0.75 & $1.37-4.75$ & $F(2,152)=5.85 p=0.003$ & 77 & $p=0.004$ & $\mathrm{~ns}(\mathrm{p}=1.0)$ \\
\hline \multirow[t]{2}{*}{ CSS NV } & 3.95 & 0.77 & $1.0-5.0$ & 4.03 & 0.66 & $2.12-5.0$ & 4.01 & 0.62 & $2.0-5.0$ & $F(2,152)=0.78 \mathrm{p}=0.46$ & 77 & & \\
\hline & & & & & & & & & & $F$-values 2-3 & & & \\
\hline IOI-AI tot & & & & 23.15 & 4.50 & $9-34$ & 22.06 & 4.99 & $9-33$ & $F(1,72)=4.59, p=0.03$ & 74 & & \\
\hline 1. Use & & & & 2.66 & 0.93 & $1-5$ & 2.61 & 0.92 & $1-5$ & $\mathrm{~F}(1,70)=0.01, \mathrm{p}=0.89$ & 74 & & \\
\hline 2. Benefit & & & & 3.08 & 1.15 & $1-5$ & 2.97 & 1.11 & $1-5$ & $F(1,71)=0.25, \mathrm{p}=0.61$ & 74 & & \\
\hline 3. RAL & & & & 2.60 & 0.93 & $1-5$ & 2.63 & 0.88 & $1-5$ & $F(1,72)=0.01, p=0.90$ & 74 & & \\
\hline 4. Satisfaction & & & & 4.27 & 1.10 & $1-5$ & 4.05 & 1.14 & $1-5$ & $F(1,72)=5.81, p=0.018$ & 74 & & \\
\hline 5. RPR & & & & 3.67 & 1.17 & $1-5$ & 3.53 & 1.13 & $1-5$ & $F(1,66)=0.82, \mathrm{p}=0.37$ & 70 & & \\
\hline 6. IoO & & & & 3.73 & 0.96 & $1-5$ & 3.57 & 0.88 & $1-5$ & $F(1,69)=1.77, \mathrm{p}=0.19$ & 72 & & \\
\hline 7. QoL & & & & 3.23 & 0.91 & $2-5$ & 3.10 & 0.84 & $2-5$ & $F(1,72)=1.91, \mathrm{p}=0.17$ & 74 & & \\
\hline $\mathrm{N}^{*}=$ The number & participan & aries c & to incomple & ete questionn & & & & & & & & & \\
\hline
\end{tabular}




\begin{tabular}{|c|c|c|c|c|c|}
\hline Effect of Age & Interaction effects & Between group effects & Post-hoc & Within group effects & Post-hoc \\
\hline CCS tot & & $F(1,75)=5.92, p=.017$ & ns & $F(2,150)=3.42, p=.035$ & ns \\
\hline CSS V & & & & $F(2,150)=4.06, p=.019$ & $p<.05$ \\
\hline HHIE tot & $F(2,148)=3.93, p=.021$ & $F(1,74)=4.82, p=.031$ & $\begin{array}{l}\text { short } p<.01 \\
\text { long } p<.001\end{array}$ & & \\
\hline HHIE E & & $F(1,74)=4.61, p=.035$ & $\mathrm{p}<.001$ & & \\
\hline HHIE S & $F(2,148)=3.70, p=.026$ & & $\begin{array}{l}\text { short } p<.05 \\
\text { long } \mathrm{p}<.001\end{array}$ & & \\
\hline Effects of Gender & Interaction effects & Between group effects & Post-hoc & Within group effects & Post-hoc \\
\hline CCS tot & & & & $F(2,150)=4.21, p=.016$ & ns \\
\hline CSS V & & & & $F(2,150)=5.9, p=.003$ & ns \\
\hline HHIE tot & & & & $F(2,148)=12.8, p<.001$ & $\begin{array}{l}p<.001 \text { women } \\
\mathrm{p}<.05 \text { men }\end{array}$ \\
\hline HHIE E & & & & $F(2,148)=10.7, p<.001$ & $p<.01$ \\
\hline HHIE S & & & & $F(2,148)=11.0, p<.001$ & $p<.01$ \\
\hline Effects of Hearing Loss & Interaction effects & Between group effects & Post-hoc & With-in group effects & Post-hoc \\
\hline CSS V & & $F(3,73)=3.92, p=.011$ & $p<.05$ & & \\
\hline HHIE tot & $F(6,144)=2.30, p=.037$ & $F(3,72)=4.85, p=.003$ & $p<.05$ & $F(2,144)=20.52, p<.001$ & $p<.001$ \\
\hline HHIE E & & $F(3,72)=2.88, p=.041$ & ns & $F(2,144)=14.91, p<.001$ & $p<.05$ \\
\hline HHIE S & $F(6,144)=2.92, p=.010$ & $F(3,72)=6.63, p=.001$ & $p<.01$ & $F(2,144)=20.16, p<.001$ & $p<.001$ \\
\hline IOI-AI & & $F(3,66)=3.21, p=.031$ & ns & & \\
\hline Effects of Region & Interaction effects & Between group effects & Post-hoc & Within group effects & Post-hoc \\
\hline CCS tot & & & & $F(2,140)=4.36, p=.014$ & ns \\
\hline CSS V & & & & $F(2,140)=5.15, p=.007$ & ns \\
\hline HHIE tot & & & & $F(2,138)=13.07, p<.001$ & ns \\
\hline HHIE E & & & & $F(2,138)=10.28, p<.001$ & ns \\
\hline HHIE S & & & & $F(2,138)=11.80, p<.001$ & ns \\
\hline Effects of SO & Interaction effects & Between group effects & Post-hoc & Within group effects & Post-hoc \\
\hline CSS V & & & & $F(2,150)=4.73, p=.010$ & ns \\
\hline HHIE tot & & & & $F(2,148)=12.14, p<.001$ & $p<.05$ yes $^{*}, p<.001$ no \\
\hline HHIE E & & & & $F(2,148)=10.16, p<.001$ & $p<.01$ no \\
\hline HHIE S & & & & $F(2,148)=10.05, p<.001$ & $p<.01$ no \\
\hline IOI-AI & $F(1,68)=6.21, p=.015$ & & ns & & \\
\hline \multicolumn{2}{|c|}{ * with attending $\mathrm{SO}, \mathrm{no}=$ without $\mathrm{SO}$} & & & & \\
\hline
\end{tabular}


Table 4. Means and SDs for the statistically significant outcome differences between age, gender, HL and attendance of SO.

\begin{tabular}{|c|c|c|c|c|c|c|c|c|c|c|c|c|}
\hline $\begin{array}{c}\text { Questionnaire } \\
\text { Age }\end{array}$ & $\begin{array}{l}\text { Mean pre } \\
\text { Younger }\end{array}$ & SD & $\begin{array}{c}\text { Mean pre } \\
\text { Older }\end{array}$ & SD & $\begin{array}{c}\text { Mean } \\
\text { post 3wk } \\
\text { Younger }\end{array}$ & SD & $\begin{array}{c}\text { Mean } \\
\text { post 3wk } \\
\text { Older }\end{array}$ & SD & $\begin{array}{c}\text { Mean } \\
\text { post } 6 \text { mo } \\
\text { Younger }\end{array}$ & SD & $\begin{array}{c}\text { Mean } \\
\text { post } 6 \text { mo } \\
\text { Older }\end{array}$ & SD \\
\hline HHIE tot & 56.00 & 14.96 & 49.78 & 19.17 & 60.18 & 14.81 & 44.31 & 18.26 & 56.18 & 18.64 & 41.57 & 18.43 \\
\hline HHIE S & 28.36 & 6.50 & 26.67 & 10.40 & 31.45 & 6.13 & 24.18 & 9.84 & 28.55 & 8.67 & 22.09 & 9.02 \\
\hline HHIE E & 27.63 & 10.27 & 23.12 & 10.86 & 28.72 & 10.44 & 20.12 & 10.48 & 27.63 & 11.62 & 19.48 & 10.78 \\
\hline CSS V & 2.59 & 0.58 & 2.94 & 0.78 & 2.83 & 0.50 & 3.20 & 0.81 & 3.01 & 0.66 & 3.10 & 0.77 \\
\hline Gender & Women & & Men & & Women & & Men & & Women & & Men & \\
\hline HHIE tot & 51.78 & 19.88 & 48.93 & 16.80 & 47.23 & 18.94 & 45.58 & 18.27 & 44.17 & 18.97 & 42.90 & 19.50 \\
\hline HHIE S & 26.76 & 10.33 & 27.13 & 9.42 & 24.68 & 9.53 & 26.13 & 10.11 & 22.55 & 8.84 & 23.80 & 9.87 \\
\hline HHIE E & 25.02 & 11.09 & 21.80 & 10.28 & 22.55 & 11.01 & 19.45 & 10.47 & 21.61 & 11.42 & 19.10 & 10.86 \\
\hline SO & With SO & & Without SO & & With SO & & Without SO & & With SO & & Without SO & \\
\hline HHIE tot & 51.47 & 21.24 & 50.41 & 17.95 & 45.05 & 19.82 & 47.12 & 18.30 & 42.42 & 21.39 & 44.10 & 18.39 \\
\hline HHIE S & 26.84 & 9.98 & 26.93 & 9.99 & 23.79 & 10.66 & 25.72 & 9.43 & 22.21 & 9.35 & 23.30 & 9.22 \\
\hline HHIE E & 24.63 & 12.85 & 23.48 & 10.19 & 21.26 & 10.61 & 21.40 & 11.01 & 20.21 & 12.71 & 20.80 & 10.78 \\
\hline HL & Mild HL & & Profound HL & & Mild HL & & Profound HL & & Mild HL & & Profound HL & \\
\hline HHIE tot & 38.0 & 19.49 & 60.00 & 22.65 & 35.56 & 18.80 & 53.71 & 17.03 & 32.22 & 16.21 & 42.86 & 20.97 \\
\hline HHIE S & 20.11 & 9.66 & 35.00 & 10.47 & 19.67 & 10.27 & 32.85 & 9.37 & 17.55 & 8.42 & 23.71 & 9.37 \\
\hline HHIE E & 17.89 & 10.53 & 25.00 & 14.65 & 15.89 & 9.24 & 20.86 & 11.99 & 14.67 & 8.78 & 19.14 & 12.56 \\
\hline CSS V & 2.39 & 0.62 & 3.50 & 0.84 & 2.80 & 0.72 & 3.36 & 1.17 & 2.67 & 0.61 & 3.30 & 0.89 \\
\hline
\end{tabular}




\begin{tabular}{|l|c|c|c|c|}
\hline Table 5. Results for the categorized COSI goals & & & & \\
\hline COSI-goals & $\begin{array}{c}\text { Number } \\
\text { of goals }\end{array}$ & Mean & SD & Range \\
\hline All goals & 212 & 3.49 & 1.04 & $1-5$ \\
\hline Learn communication skills & 87 & 3.18 & 0.93 & $1-5$ \\
\hline $\begin{array}{l}\text { Learn about hearing aids and assistive listening } \\
\text { devices }\end{array}$ & 39 & 3.69 & 1.16 & $2-5$ \\
\hline Meet in a group to share experiences & 37 & 3.76 & 1.04 & $1-5$ \\
\hline Learn more about my hearing loss & 24 & 3.67 & 0.92 & $2-5$ \\
\hline Prevent isolation and increase self-confidence & 14 & 4.07 & 1.00 & $2-5$ \\
\hline Learn how to hear better on the telephone & 8 & 3.12 & 1.13 & $2-5$ \\
\hline Learn how to hear better to TV and in the car & 3 & 3.67 & 1.53 & $2-5$ \\
\hline \multicolumn{2}{|l|}{} \\
\hline Note: A mean of 2 means describing the goal as "no different", 3 "slightly better", & \\
\hline 4 "better" and 5 means "much better". & & & \\
\hline
\end{tabular}


Table 6. Overview of statistically significant overall and between group outcomes for ACE-studies

\begin{tabular}{|c|c|c|c|}
\hline & Present study & $\begin{array}{l}\text { Öberg et al. } \\
\text { 2014a }\end{array}$ & $\begin{array}{c}\text { Hickson et al. } \\
2007 \mathrm{a}\end{array}$ \\
\hline \multicolumn{4}{|l|}{$\begin{array}{l}\text { Outcome measure } \\
\text { Overall }\end{array}$} \\
\hline Activity and participation ${ }^{a}$ & Yes & Yes & Yes \\
\hline Mental health ${ }^{\text {b }}$ & No & Yes & Yes \\
\hline \multicolumn{4}{|l|}{ Age } \\
\hline Activity and participation & Older + & No & No \\
\hline Mental health & No & No & No \\
\hline \multicolumn{4}{|l|}{ Gender } \\
\hline Activity and participation & Women+ Men+ & Women + & No \\
\hline Mental health & No & Women + & No \\
\hline Communication strategies & No & Women + & No \\
\hline \multicolumn{4}{|l|}{ Significant Other } \\
\hline Activity and participation & Without SO+ & $\mathrm{n} / \mathrm{a}$ & No \\
\hline Mental health & No & $\mathrm{n} / \mathrm{a}$ & No \\
\hline Communication strategies & No & $\mathrm{n} / \mathrm{a}$ & No \\
\hline \multicolumn{4}{|l|}{ Hearing Loss } \\
\hline Activity and participation & Profound HL+ & Mild HL + & No \\
\hline Mental health & No & Moderate HL+ & No \\
\hline Communication strategies & No & Mild HL + & No \\
\hline
\end{tabular}

+ explain improved group

${ }^{a}$ Measured with the Hearing Handicap Inventory for the Elderly in present and Öberg et al. and with the Hearing Handicap Questionnaire and Quantified Denver Scale of Communicative Function, in Hickson et al.

${ }^{\mathrm{b}}$ Measured with the Hospital and Depression Scale in present and Öberg et al. and with the Ryff Psychological Well-Being Scale, in Hickson et al.

${ }^{c}$ Measured with the Communication Strategies Scale in present and

Öberg et al. and with the Self-Assessment of Communication, in Hickson et al. 